\title{
CONDUCTING SYSTEMATIC LITERATURE REVIEWS IN INFORMATION SYSTEMS: AN ANALYSIS OF GUIDELINES
}

\author{
Zefeng Bai, Bentley University, zbai@bentley.edu \\ Nitin Jain, BentleyUniversity, njain@bentley.edu \\ Roman Kurdyukov, Bentley University, rkurdyukov@bentley.edu \\ JoannaWalton, Bentley University,jwalton@bentley.edu \\ YingWang, Bentley University,ywang@bentley.edu \\ TylerWasson, Bentley University, twasson@bentley.edu \\ Xiaolin Zhu, Renmin University of China, zhuxiaolin@ruc.edu.cn \\ Alina M. Chircu, BentleyUniversity, achircu@bentley.edu
}

\begin{abstract}
This paper analyzes the guidelines for systematic literature reviews in the Information Systems (IS) field based on 12 influential journal articles published from 2002 through 2018. The analysis indicates that there is heterogeneity among the selected articles in terms of defining the purposes of a literature review, but that in general there is homogeneity among the guidelines provided by various authors regarding conducting the review. The findings also highlight a temporal evolution of the guidelines, the emergence of critiques of too much systematization, and the proposal of new approaches for conducting literature reviews. The number of times the selected papers are cited each year increases with time, and the guidelines are cited in a diversity of fields, suggesting the existence of a growing interest in conducting systematic literature reviews among scholars in IS and other disciplines. The results of the analysis can help guide researchers from IS and other fields to understand not just the general best practices for conducting literature reviews, but also the emerging options available for generating a quality literature review paper.
\end{abstract}

Keywords: Information Systems (IS), Systematic Literature Review (SLR) Best Practices

\section{INTRODUCTION}

A literature review is a critical endeavor in academic research that can summarize the main theoretical underpinnings and empirical findings in a particular academic field, uncover potential research avenues, and develop new theories all with the goal of advancing knowledge (Webster and Watson, 2002). A systematic literature review is a particular type of review which emphasizes rigorous, explicit, and reproducible methods (Moher et al., 2009; Pickering and Byrne, 2014; Tranfield et al., 2003). It enables any researchers to perform a "critical summary and assessment of the range of existing materials dealing with knowledge and understanding in a given field" (Rowe, 2014), thus providing a firm foundation for a research topic and methodology and demonstrating that the proposed research contributes new knowledge (Levy and Ellis, 2006). Indeed, a well-conducted literature review is the type of paper "that every researcher looks for when starting a research study" (Rowe, 2014). While it benefits all researchers, conducting a systematic literature review may be particularly useful for early career researchers, including PhD students, who can publish the literature review part of a larger research project as a stand-alone paper (Pickering and Byrne, 2014).

Various guidelines for systematic literature reviews have been developed in many disciplines, including healthcare, management, software engineering, environmental sciences and geography (Kitchenham et al., 2009; Moher et al., 2009; Pickering and Byrne, 2014; Tranfield et al., 2003). Following this trend, researchers in the information systems (IS) field have produced important studies about conducting literature reviews in a systematic way. In this paper, we compare and contrast these studies, summarizing the proposed guidelines and analyzing their evolution and impact over time. Our research focuses on the following research questions:

1. What is the purpose of a systematic literature review in the IS field?

2. What are the best practice guidelines for conducting a systematic literature review in the IS field?

3. How do the best practice recommendations evolve over time?

4. What impact do the systematic literature review guidelines have on the IS and other fields? 


\section{METHODOLOGY}

We adopted a structured approach (Webster and Watson, 2002) for selecting articles that describe guidelines for conducting literature reviews, in general, and SLRs, in particular, in the IS field. We searched for articles published in leading journals (as defined by the IS community and by ranking lists). To ensure we identify all the relevant articles, we employed several strategies. First, we conducted a search for relevant terms (literature review) in the title, abstract and keywords of articles indexed in EBSCO Business Search Complete and Web of Science (two well-known research indexing services), limiting the search to peer-reviewed scholarly articles in journals that include "information" (as a component of information systems) in their name. We repeated this search in Google Scholar (the largest web search engine for scholarly literature), focusing on keywords that occur only in the title of the article. Second, we selected for analysis only papers published in reputable journals for analysis. We used the Australian Business Deans Council (ABDC) rankings list as a guideline (selecting only A*, i.e. top 5-7\%, journals and A, i.e. next $15-25 \%$, journals). Third, we analyzed the article abstracts and, if necessary, the full text, and selected only the articles that focus on systematic literature review definition, guidelines or best practices. Fourth, we performed forward as well as backward searches to identify other articles we need to consider. We considered any articles that seemed relevant if they met most of the criteria outlined above and were also highly cited. This resulted in the inclusion of an article from a journal that was not ranked by ABDC but was cited by other articles published in top journals. We also included two editorials and one guest editorial because they set the direction for literature reviews submissions to top journals. However, we did not select any editorially-reviewed papers (such as those presenting the results of conference panel discussions) or short invited commentaries to peer-reviewed articles.

At the end of this structured search process, we identified 12 articles for our study, published from 2002 through 2018. For each article, we collected citation data from Google Scholar (which includes citations in a broad range of publications - journals, conference proceedings, research reports, etc.) and Web of Science Core Collection (which includes citations in a list of journals selected based on quality, and thus provides smaller citation numbers than Google Scholar). We also collected information about the ranking of the journal where the articles were published (using the ABDC list). Table 1 presents a summary of this information (see Table 1).

Table 1. List of Articles Selected for This Study Notes: ${ }^{e}$ denotes editorials/guest editorials; citation numbers were collected in February 2019

\begin{tabular}{|c|c|c|c|c|}
\hline $\begin{array}{c}\text { Year of } \\
\text { Publication }\end{array}$ & Articles & Journal [ABDC Ranking] & $\begin{array}{c}\text { Google } \\
\text { Scholar } \\
\text { Citations }\end{array}$ & $\begin{array}{c}\text { Web of } \\
\text { Science } \\
\text { Citations }\end{array}$ \\
\hline 2002 & Webster and Watson (2002) & MIS Quarterly (MISQ) $[A *]$ & 5151 & 1869 \\
\hline 2006 & Levy and Ellis (2006) & Informing Science Journal [NA] & 1071 & 234 \\
\hline 2013 & Wolfswinkel et al. (2013) & $\begin{array}{l}\text { European Journal of Information Systems } \\
\text { (EJIS) }\left[A^{*}\right]\end{array}$ & 417 & 95 \\
\hline 2014 & Rowe $(2014)^{\mathrm{e}}$ & EJIS $\left[A^{*}\right]$ & 220 & 65 \\
\hline \multirow[t]{7}{*}{2015} & $\begin{array}{l}\text { Boell and Cecez- } \\
\text { Kecmanovic (2014) }\end{array}$ & $\begin{array}{l}\text { Communications of the Association for } \\
\text { Information Systems (CAIS) }[A]\end{array}$ & 217 & 53 \\
\hline & Okoli (2015) & CAIS $[A]$ & 82 & 19 \\
\hline & Schryen (2015) & CAIS $[A]$ & 54 & 9 \\
\hline & Templier and Paré (2015) & CAIS $[A]$ & 57 & 16 \\
\hline & Bandara et al. (2015) & CAIS $[A]$ & 72 & 19 \\
\hline & $\begin{array}{l}\text { Boell and Cecez- } \\
\text { Kecmanovic (2015) }\end{array}$ & $\begin{array}{l}\text { Journal of Information Technology (JIT) } \\
{\left[A^{*}\right]}\end{array}$ & 141 & 58 \\
\hline & Paré et al. (2015) & Information \& Management (I\&M) $\left[A^{*}\right]$ & 236 & 83 \\
\hline 2018 & Leidner $(2018)^{\mathrm{e}}$ & $\begin{array}{l}\text { Journal of the Association for Information } \\
\text { Systems (JAIS) }\left[A^{*}\right]\end{array}$ & 2 & 0 \\
\hline
\end{tabular}




\section{Issues in Information Systems}

Volume 20, Issue 3, pp. 83-93, 2019

\section{ANALYSIS AND FINDINGS}

In this section, we present the results of our in-depth analysis of the content of the articles. We first describe the sample from a temporal and geographical perspective. Next, we provide an overview of the purposes and types of reviews. We then present a detailed examination of literature review guidelines, focusing on the core elements of the literature review process identified by Webster and Watson (2002) - the earliest and the most highly cited article in our sample.

\section{Temporal and Geographical Analysis}

As shown in Table 1, the first - and most cited - article focusing on IS literature review guidelines was published as an MISQ guest editorial in 2002. Another highly cited article followed in 2006, after which there was a significant gap - until 2013 - in publications. Interest in the topic seems to peak in recent years, with 10 articles being published since 2013. The high number of articles published in 2015 is due in part to a CAIS special issue on the literature review in information systems. However, two 2015 articles appeared in other publications as well. The last relevant article was published in 2018 as another editorial. Most article authors are based in Europe (4 articles), Australia (4 articles), Canada ( 3 articles), and the United States ( 2 articles). Most articles are originating from the same geography: four articles authored by a single researcher, seven articles co-authored by teams of 2-4 researchers (from the same geography), and only one article co-authored by a team of researchers from Australia, Germany, Austria, and Malaysia. In fact, barring Malaysia, no other Asian university is represented. This indicates that there is potential for researchers in other parts of the world to contribute their insights to how literature review guidelines may need to be modified based on geographical differences (especially related to publishing in different languages or using different philosophical perspectives on research).

\section{Literature Review Purposes and Types}

A literature review can be a part of a research paper, a section in a thesis or in a project proposal, or a standalone review (Schryen, 2015). With increasing attention to the importance of the literature review, more and more studies have been done on how to conduct a stand-alone review systematically - i.e. how to produce a quality "journal-length paper that reviews the literature in a field without the author's collecting or analyzing any primary data" (Okoli, 2015). Webster and Watson (2002) define what such a review paper ought to do: create a foundation for advancing knowledge, facilitate theory development, summarize research fields and uncover potential research avenues. Levy and Ellis (2006) list essential characteristics of a review paper: analyze and synthesize quality literature in a systematic way, provide a firm foundation for a research topic and methodology, and demonstrate that the proposed research contributes new knowledge.

Most of the papers we analyzed for this study agree that well-constructed literature reviews are a useful tool for understanding the body of knowledge in a particular field (Schryen 2015; Templier and Paré 2015), examining a field's recent or current literature via various theoretical levels to frame and support a research project (Bandara et al. 2015), identifying gaps in the literature (Rowe 2014; Okoli 2015), surfacing new research problems and interesting research questions (Boell and Cecez-Kecmanovic 2014, 2015), as well as for improving public policy and guiding the world of practice (Okoli 2015; Boell and Cecez-Kecmanovic 2015). The full list of the stated purposes of a literature review is presented in Table 2 .

A few of the papers we analyzed also include an explicit discussion of types of literature reviews. For example, Paré et al. (2015) develop a typology of nine ideal types: narrative, descriptive, scoping, qualitative systematic, metaanalytic, realist, umbrella, theoretical, and critical. More recently, Leidner (2018) develops an alternative typology in response to Paré and colleagues since she perceived a pressure from journal reviewers and editors to confine one's literature review to one of the narrow types. In her polylithic typology, reviews are classified based upon research objective (which ranges from synthesizing to theorizing) and focus (which ranges from describing to identifying trends/gaps), resulting in four partially overlapping categories: broad theorizing, specific theorizing, organizing, and assessing reviews.

We find that each paper in our sample chooses to emphasize a slightly different purpose for a literature review, and while some papers tend to agree with others regarding the usefulness of such reviews for understanding prior research and developing new knowledge, there are also many differences among papers. This diversity of opinions may be confusing for researchers - especially early career ones - and for reviewers tasked to evaluate such papers. Despite the plethora of specific guidelines, conducting a quality literature review remains an art - and the onus is on authors 
to produce a review that is "not so new and different so as to render the past unrecognizable, but new and different enough to render the future imaginable" (Leidner, 2018).

Table 2. Primary Purposes of Literature Reviews

\begin{tabular}{|l|l|}
\hline \multicolumn{1}{|c|}{ Article } & \multicolumn{1}{c|}{ Stated Purpose } \\
\hline $\begin{array}{l}\text { Webster and Watson } \\
(2002)\end{array}$ & $\begin{array}{l}\text { To provide a firm foundation for advancing knowledge, developing new theory, } \\
\text { summarizing a research field, and finding new research directions }\end{array}$ \\
\hline Levy and Ellis (2006) & $\begin{array}{l}\text { To use valid research methodologies and approaches to map and provide a firm } \\
\text { foundation to add to a field's body of knowledge, and to build and test hypotheses }\end{array}$ \\
\hline Wolfswinkel et al. (2013) & To build a foundation for theory development \\
\hline Rowe (2014) & To gain an understanding of a particular research stream \\
\hline $\begin{array}{l}\text { Boell and Cecez- } \\
\text { Kecmanovic (2014) }\end{array}$ & To understand a field's current body of knowledge in order to advance research \\
\hline Okoli (2015) & To summarize academic work for other academics to inform policy and practice \\
\hline Schryen (2015) & To provide guidance for academics \\
\hline Templier and Paré (2015) & $\begin{array}{l}\text { Varied (to summarize previously published research, to provide new } \\
\text { conceptualizations, models, theories or methodological approaches, to map bodies of } \\
\text { literature and draw conclusions, to test hypotheses based on prior findings) }\end{array}$ \\
\hline Bandara et al. (2015) & To create a firm foundation for advancing knowledge \\
\hline $\begin{array}{l}\text { Boell and Cecez- } \\
\text { Kecmanovic (2015) }\end{array}$ & To advance knowledge that improves policy and practice \\
\hline Paré et al. (2015) & $\begin{array}{l}\text { Varied (to summarize the literature in a field, to aggregate previous findings, to } \\
\text { build explanations, to critically analyze the literature to reveal weaknesses, } \\
\text { contradictions, controversies or inconsistencies) }\end{array}$ \\
\hline Leidner (2018) & Varied (from synthesizing the literature to theorizing from the literature) \\
\hline
\end{tabular}

\section{Literature Review Guidelines - General Stages}

Most articles in our sample explicitly list the stages associated with creating a literature review. Table 3 provides a summary of these stages. Most authors discuss conducting a literature review as a highly structured, sequential process comprising several steps.

Table 3. General Stages of a Literature Review

\begin{tabular}{|c|c|c|c|c|c|c|c|}
\hline $\begin{array}{l}\text { Levy and } \\
\text { Ellis (2006) }\end{array}$ & $\begin{array}{c}\text { Wolfs- } \\
\text { winkel et } \\
\text { al. (2013) }\end{array}$ & $\begin{array}{c}\text { Boell and } \\
\text { Cecez- } \\
\text { Kecmanovic } \\
(\mathbf{2 0 1 4}) \\
\end{array}$ & Okoli (2015) & Schryen (2015) & $\begin{array}{c}\text { Templier and Paré } \\
\text { (2015) }\end{array}$ & $\begin{array}{l}\text { Bandara et } \\
\text { al. (2015) }\end{array}$ & $\begin{array}{c}\text { Boell and } \\
\text { Cecez- } \\
\text { Kecmanovic } \\
(2015) \\
\end{array}$ \\
\hline $\begin{array}{l}1 \text { Inputs } \\
2 \text { Processing } \\
\text { (iterative } \\
\text { process) } \\
3 \text { Outputs }\end{array}$ & $\begin{array}{l}\text { Iterative } \\
\text { process } \\
1 \text { Define } \\
2 \text { Search } \\
3 \text { Select } \\
4 \text { Analyze } \\
5 \text { Present }\end{array}$ & $\begin{array}{l}\text { Mutually- } \\
\text { intertwined } \\
\text { hermeneutic } \\
\text { circles } \\
\text { Search and } \\
\text { acquisition } \\
\& \\
\text { Analysis and } \\
\text { interpretation }\end{array}$ & $\begin{array}{l}1 \text { Planning } \\
2 \text { Selection } \\
3 \text { Extraction } \\
4 \text { Execution }\end{array}$ & $\begin{array}{l}1 \text { Framing } \\
2 \text { Search and } \\
\text { Assessment } \\
3 \text { Synthesis } \\
4 \text { Interpretation } \\
5 \text { Guidance } \\
6 \text { Conclusions }\end{array}$ & $\begin{array}{l}\text { Iterative process } \\
1 \text { Formulating problem } \\
2 \text { Searching } \\
3 \text { Screening for } \\
\text { inclusion } \\
4 \text { Assessing quality } \\
5 \text { Extracting } \\
6 \text { Analyzing and } \\
\text { synthesizing }\end{array}$ & $\begin{array}{l}\text { Iterative } \\
\text { process } \\
1 \text { Extract } \\
2 \text { Organize } \\
\text { and prepare } \\
3 \text { Code and } \\
\text { analyze } \\
4 \text { Write-up } \\
\text { and present }\end{array}$ & $\begin{array}{l}1 \text { Establish } \\
\text { review protocol } \\
2 \text { Search } \\
3 \text { Select } \\
4 \text { Summarize } \\
\text { evidence } \\
5 \text { Disseminate } \\
\text { results }\end{array}$ \\
\hline
\end{tabular}

There is general agreement that the first step involves determining the focus of the review and planning an appropriate course of action (described as defining, framing, planning, formulating the problem, or establishing the review protocol). Different authors provide varying levels of detail regarding the intermediate steps. These include the literature search step (define in most papers separately as searching, or as part of the selection/extraction process), the articles selection step (defined as selecting, extracting, acquiring, screening for inclusion \& assessing quality \& extracting, assessing, or organizing and preparing), and the analysis and interpretation step (defined as analyzing and interpreting or synthesizing, executing, coding and analyzing, or summarizing evidence). Some guidelines also include a final step - presenting the results (defined as presenting, writing up and presenting, discussing guidance and conclusions, and disseminating the results). We discuss these major steps in detail in the next sections. 


\section{Issues in Information Systems \\ Volume 20, Issue 3, pp. 83-93, 2019}

Overall, while the exact descriptions of the steps may vary, most guidelines agree on the general stages which comprise the process of doing a literature review, and these stages seem to remain stable as new guidelines are published over time. In addition, many guidelines recognize the iterative nature of the process (where steps can be repeated to add new papers, for example). Note however that there is a distinction between the linear, formal, protocol-based view of process steps promoted by most guidelines and the hermeneutic view, which views the steps as two intertwined circles that build recursively on each other and create knowledge through subjective interpretation and understanding (Boell and Cecez-Kecmanovic, 2014). The emerging recommendation is to not overly-mechanize the process, but instead choose the type of review (and level of detail) that can help authors explore the literature (by evolving the objective, focus, and topic of the review) and challenge existing assumptions in order to generate novel, unexpected insights (Leidner, 2018).

\section{Guidelines - Identifying Relevant Literature}

The earliest article in our sample (Webster and Watson, 2002) discussed the ideal search criteria for identifying relevant literature. Over the years, additional attention was given to defining inclusion/exclusion criteria and the means of assessing the quality of research (see Table 4). All papers discuss the use of electronic databases, with Templier and Paré (2015) uniquely highlighting the impact of searching manually through materials. Conference proceedings were mentioned by many as an important source of primary studies, but with the reservation that they may have lower impact and quality (Bandara et al. 2015). Keyword searches, searching in literatures belonging to different fields of study, and searching forward for citing articles and backward for cited articles were also frequent topics. Roughly half of the papers mentioned stopping rules, meaning that there is a certain point of saturation, depending on the purposes and scope of the review, where finding additional articles may not add value to the review.

In addition, Webster and Watson (2002) provide explicit guidelines for determining the boundaries of a review, which included level of analysis, temporal/contextual limits, scope of review, assumptions, and target audience. Later articles emphasized the selection of relevant literature as the key boundary-setting activity. While there is obviously considerable overlap between literature identification and boundary setting, as both involve determining the level of analysis and the scope of the review, it can be argued that the later papers in our sample could have benefitted from a more explicit discussion of determining the boundaries of a literature review.

Boell and Cecez-Kecmanovic (2015) provide a somewhat different perspective on the literature search process that is critical of many common search criteria. For example, they caution against the assumption that good search terms can be found, that search terms can identify all relevant documents, and that electronic databases provide good coverage of the relevant literature. It should be noted, however, that many other authors discuss the limitations to using only one search method and the need to search multiple sources.

Table 4. Literature Search Guidelines

\begin{tabular}{|l|l|}
\hline \multicolumn{1}{|c|}{ Search Strategy } & \multicolumn{1}{c|}{ Articles } \\
\hline Electronic databases & $\begin{array}{l}\text { Webster and Watson (2002); Levy and Ellis (2006); Wolfswinkel et al. (2013); } \\
\text { Rowe (2014); Boell and Cecez-Kecmanovic (2014); Okoli (2015); Schryen } \\
\text { (2015); Templier and Paré (2015); Bandara et al. (2015) }\end{array}$ \\
\hline Search materials in person & Templier and Paré (2015) \\
\hline Conference proceedings & $\begin{array}{l}\text { Webster and Watson (2002); Wolfswinkel et al. (2013); Boell and Cecez- } \\
\text { Kecmanovic (2014); Okoli (2015); Schryen (2015); Templier and Paré (2015); } \\
\text { Bandara et al. (2015) }\end{array}$ \\
\hline Keyword searches & $\begin{array}{l}\text { Levy and Ellis (2006); Wolfswinkel et al. (2013); Rowe (2014); Boell and } \\
\text { Cecez-Kecmanovic (2014); Okoli (2015); Schryen (2015); Templier and Paré } \\
\text { (2015); Bandara et al. (2015) }\end{array}$ \\
\hline $\begin{array}{l}\text { Interdisciplinary search within } \\
\text { and outside of the field }\end{array}$ & $\begin{array}{l}\text { Webster and Watson (2002); Rowe (2014); Boell and Cecez-Kecmanovic (2014); } \\
\text { Okoli (2015); Schryen (2015); Templier and Paré (2015); Bandara et al. (2015) }\end{array}$ \\
\hline Forward and backward searches & $\begin{array}{l}\text { Webster and Watson (2002); Levy and Ellis (2006); Wolfswinkel et al. (2013); } \\
\text { Boell and Cecez-Kecmanovic (2014); Okoli (2015); Schryen (2015); Templier } \\
\text { and Paré (2015); Bandara et al. (2015) }\end{array}$ \\
\hline Stopping rule & $\begin{array}{l}\text { Levy and Ellis (2006); Boell and Cecez-Kecmanovic (2014); Okoli (2015); } \\
\text { Schryen (2015) }\end{array}$ \\
\hline
\end{tabular}




\section{Issues in Information Systems \\ Volume 20, Issue 3, pp. 83-93, 2019}

\section{Guidelines - Inclusion Criteria and Assessing Quality}

While many authors simply discuss creating search criteria based on the topic of interest, Webster and Watson (2002) emphasize the importance of identifying major contributions by looking at leading journals, and then expanding the article set by doing backward and forward searches. Boell and Cecez-Kecmanovic (2015) highlight the need to include only literature that has high quality and demonstrates validity in its findings. A common suggestion made by the articles we analyzed was that the quality of a piece of research may be judged by assessing the quality of the publication in which it appears. This can be done by using well-known journal rankings lists (Levi and Ellis, 2006). While most authors agreed that journal quality rankings are reliable, determining the quality of conference proceedings and papers is less straightforward. Therefore, Levy and Ellis (2006) suggest limiting conference proceedings to those referenced in top journals and Wolfswinkel et al. (2013) suggests being selective when including conference proceedings. In a similar vein, Bandara et al. (2015) caution that conference papers may be considered to have lower impact compared to journal articles.

\section{Guidelines - Analysis and Theoretical Development}

One major analysis recommendation is common among the articles in our sample - synthesizing the literature by classifying it in broad categories (Rowe, 2014). The analysis can be based on aggregating, discussing, organizing, comparing and critiquing the articles selected for review (Okoli, 2015). Coding and concept-centric analysis are important tools for literature synthesis. Coding is used to either identify new concepts (not known a priori) that emerge from the selected articles (Wolfswinkel et al., 2013) or classify the articles into pre-determined categories based on the authors' understanding of existing theories, constructs and variables (Levy and Ellis, 2006). Qualitative data management tools can be used to code textual data, such as journal or conference articles (Bandara et al. 2015). Concept-centric analysis is a form of synthesizing the literature first discussed by Webster and Watson (2002) and cited by many other papers. It recommends organizing the results of the literature review based on concepts or themes rather than by authors or chronology.

The discussion of theoretical development varies significantly among the articles we analyzed. Webster and Watson (2002) seem to be the only authors that argue that a literature review should create propositions and set out a way to evaluate those propositions. In the subsequent literature, recommendations on how to develop theory are more general, emphasizing systematic critical assessment of past research and identification of gaps and potential research questions (Wolfswinkel et al. 2013; Schryen 2015; Templier and Paré 2015). Most recently, Leidner (2018) argues that some literature review papers can contribute to the body of knowledge by synthesizing the literature, without developing theory, while other literature review papers contribute by analyzing a stream of research and generating new theories about a phenomenon or about explaining a gap in the literature.

\section{Guidelines - Writing and Structure}

Many articles we reviewed mentioned several dimensions related to the writing process and the structure of the literature review report. For example, Webster and Watson (2002) comment both on the recommended structure and the tone (collegial) and tense (consistent) of the review. They also discuss how to identify areas for improvement (which will increase chances of publication) by asking the questions: "what's new?" (contribution), "so what?" (impact), "why so?" (logic), and "well done?" (thoroughness). Schryen (2015) also recommends a respectful tone, as well as synthesizing more than quoting, discussing the contributions to domain knowledge and the impact of each of the articles examined, and using visualizations such as tables, diagrams, and figures to synthesize and conceptualize the contribution. He also suggests that working with others can be beneficial; recommending multiple authorship as well as requesting "friendly reviews" from experts in the field. Okoli (2015) recommends clearly reporting the process to ensure replicability and highlighting any novel findings.

Several authors propose a specific review structure. Levy and Ellis (2006) recommend including an introduction, sections addressing independent and dependent variables and summarizing the studies that focus on those variables, and a summary. Wolfswinkel et al. (2013) recommend including an introduction and sections focused on the rationale for the review and why this type of literature review is relevant, methodology, findings and future research directions, discussion, conclusions and implications for new research options and practice, and limitations. In a similar vein, Bandara et al. (2015) recommend including an abstract and introduction as well as sections focused on methods, results, discussion and conclusions. 


\section{Issues in Information Systems}

Volume 20, Issue 3, pp. 83-93, 2019

\section{Impact Analysis}

In this section, we analyze the impact of the journal articles we used via a systematic analysis of their respective citation counts in Google Scholar and the Web of Science. Table 1 and Figures 1 and 2 show the results of this analysis.

As expected, the earliest paper in our sample (Webster and Watson, 2002) leads with the highest number of citations (5151 in Google Scholar and 1869 in Web of Science), with papers published in 2006 and 2013 being in a distant second (Levy and Ellis, 2006) and third place (Wolfswinkel et al., 2013). In addition, several papers published in 2014 and 2015 show respectable citation numbers, but the citation counts are lower for the rest of the 2015 papers. We also note that in general the number of citations is steadily increasing every year for every paper we analyzed, suggesting that the topic of systematic literature reviews is, as a whole, receiving increased attention, and that the IS perspective on how to conduct such reviews is useful to other researchers.

To better understand the impact of the papers we analyze in this study, we perform a vintage-based citation analysis. Vintage and cohort analyses are used in credit risk modeling to build acquisition and behavioral scorecards for risk and marketing models (Siddiqui, 2012). For our analysis, vintage analysis is done to understand the citation counts from the year of inception. This helps compare citation counts for articles of the same age - i.e. during the publication year (year 1), the year after publication (year 2), etc. For example, year 1 for Webster and Watson (2002) is 2002, and the year 1 citations number counts only citations by papers published in 2002, while the year 2 citation number counts only citations in 2003 articles. Similarly, year 1 for Levy and Ellis (2006) is 2006, year 2 is 2007, and so on. Therefore, vintage analysis allows us to compare citation counts in the same year after publication (i.e. for year 1, we compare 2002 citations for Webster and Watson (2002) with 2006 citations for Levy and Ellis (2006), etc.). Note that Leidner (2018) is excluded from this analysis since it only has two citations in 2018. In addition, due to space considerations, we present only the Google Scholar results (since we obtained similar results by analyzing Google Scholar and Web of Science citation counts).

Figure 1 shows that the oldest paper in our sample (Webster and Watson, 2002) received similar citation counts to other papers during the first years after publication, becoming more popular only 7-8 years after publication, after which its citation count started growing exponentially every year. The second oldest paper (Levi and Ellis, 2006) had a similar trajectory for the first eight years, but then its citations counts grew at a much slower rate. Figure 2 shows the citation counts trajectories for the select recent papers (2013-2015). Paré et al. (2015) seems to be the most impactful in this group, followed by Wolfswinkel et al. (2013), Rowe (2014) and Boell and Cecez-Kecmanovic (2014). The remaining papers, all published in 2015, have slower citations counts growth.

We also analyzed citations by document type and field/discipline, which is only possible by using Web of Science data. Citations to articles in our sample were most common in original research articles, followed by proceedings papers and reviews, which cite them in relatively equal proportion Furthermore, as indicated by the heat maps we created to analyze the concentration of citations across both articles and fields (see Figure 3), most citations for all papers come from the Computer Science / Information Systems, Information Science / Library Science, and Management field. Interestingly though, the diversity of fields citing the articles in our sample is significant and includes fields as diverse as Political Science and Psychology (Rowe, 2014), Business Finance (Webster \& Watson, 2002), Environmental Engineering and Environmental Sciences (Bandara et al., 2015; Rowe, 2014; Webster and Watson, 2012), Clinical Neurology (Wolfswinkel et al., 2013), Nursing (Bandara et al., 2015), Rehabilitation (Okoli, 2015), Social Issues (Wolfswinkel et al., 2013), and Public Administration (Okoli, 2015). In our view, this indicates the growing influence of the IS literature review guidelines on other fields, and it augurs well for the exchange of methods, approaches, and practices across fields and disciplines. 


\section{Issues in Information Systems}

Volume 20, Issue 3, pp. 83-93, 2019

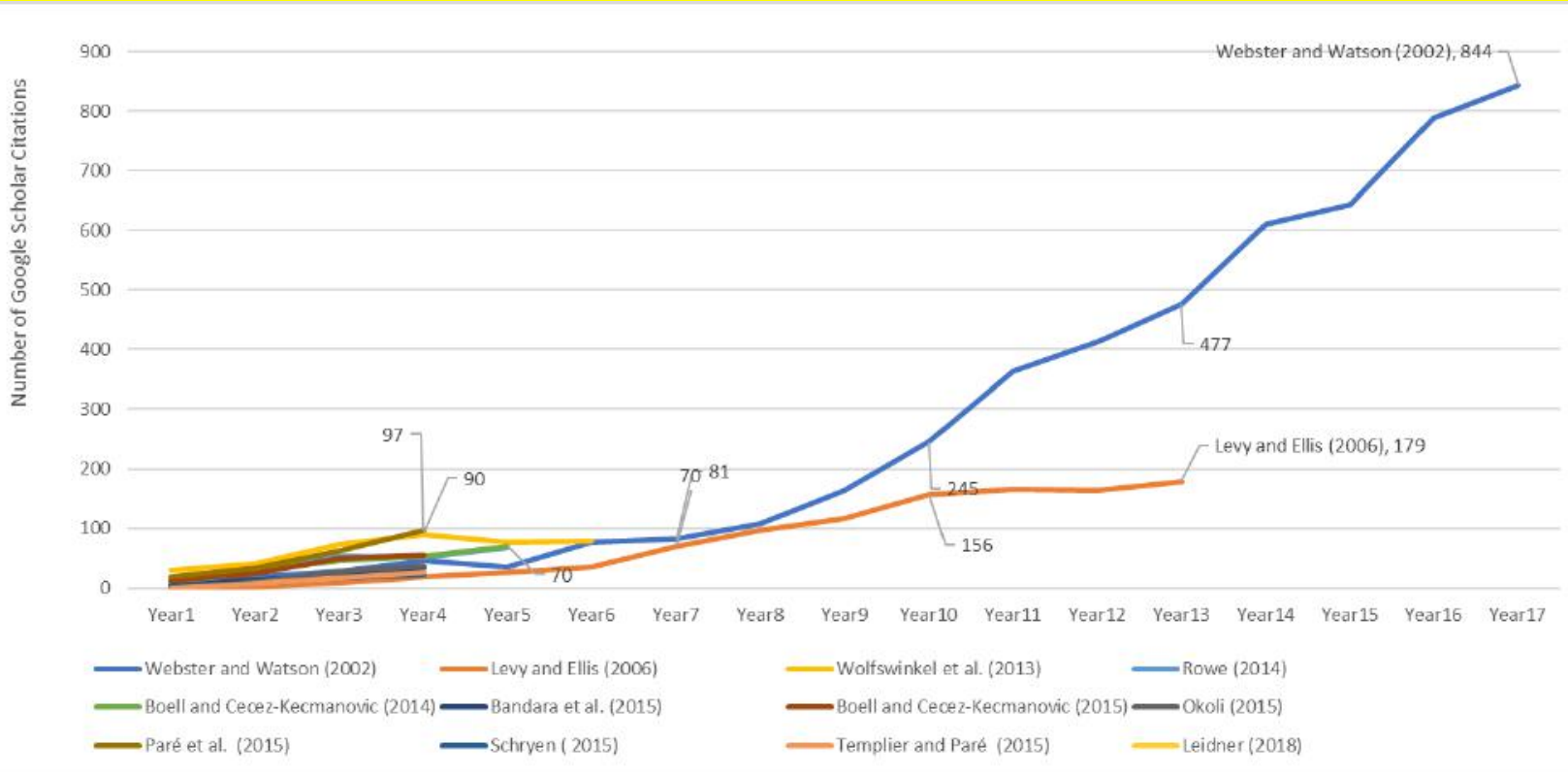

Figure 1. Citations Vintage Analysis for All Papers (2002-2018)

Note: Year $1=$ the year of publication for each paper, Year2 $=$ Year1+1, etc.

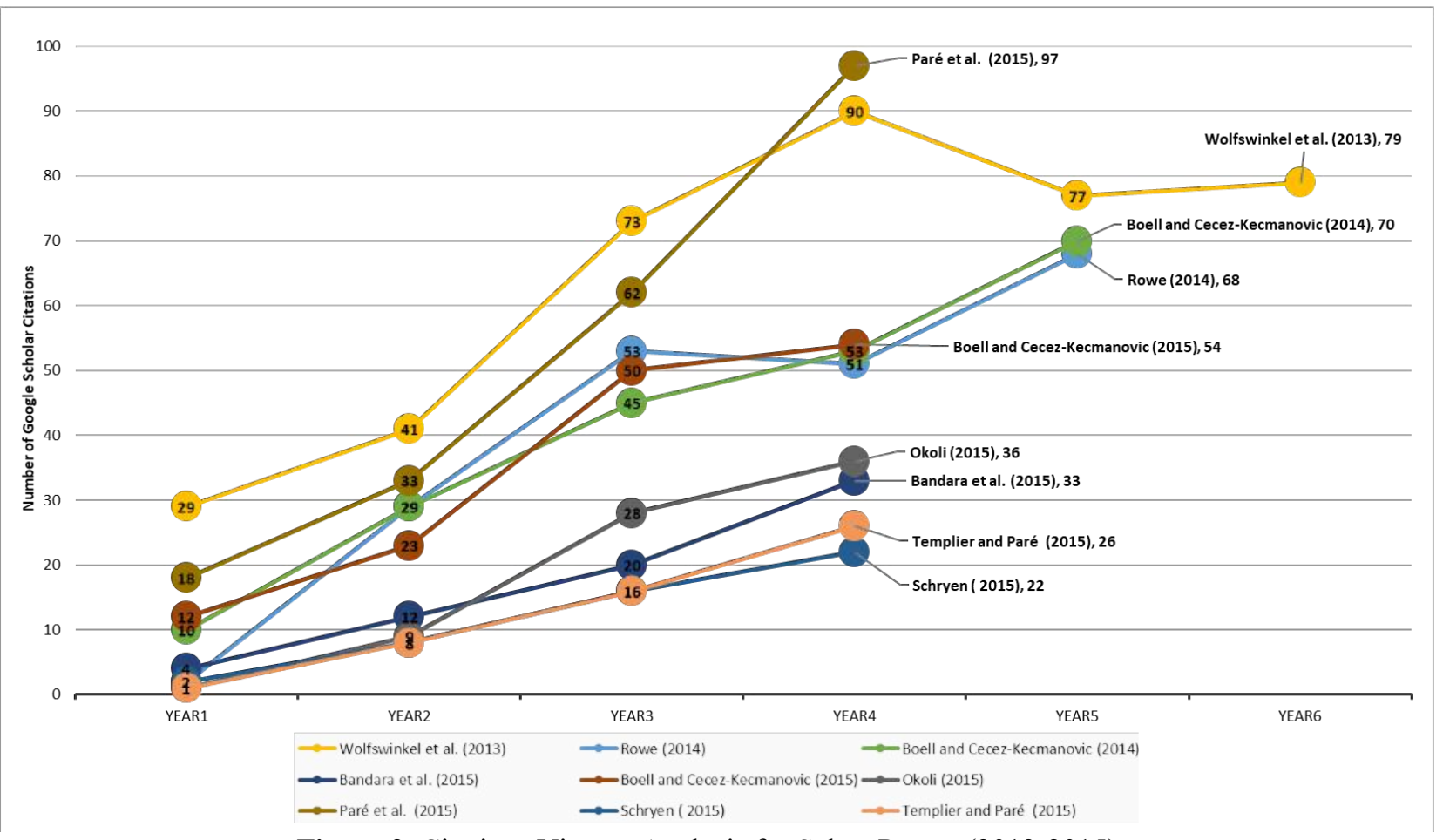

Figure 2. Citations Vintage Analysis for Select Papers (2013-2015)

Note: Yearl $=$ the year of publication for each paper, Year2 $=$ Year 1+1, etc. 


\begin{tabular}{|c|c|c|c|c|c|c|c|c|}
\hline Fields & $\begin{array}{l}\text { Webster and } \\
\text { Watson (2002) }\end{array}$ & $\begin{array}{l}\text { Wofswinkel et } \\
\text { al. (2013) }\end{array}$ & Rowe (2014) & Par'e (2015) & $\begin{array}{c}\text { Bandara et al. } \\
\text { (2015) }\end{array}$ & $\begin{array}{l}\text { Templier and } \\
\text { Par'e (2015) }\end{array}$ & Okoli (2015) & Schryen (2015) \\
\hline $\begin{array}{c}\text { Computer Science Information } \\
\text { Systems }\end{array}$ & 239 & 33 & 36 & 22 & 7 & 5 & 4 & 6 \\
\hline $\begin{array}{l}\text { Information Science Library } \\
\text { Science }\end{array}$ & 124 & 20 & 27 & 18 & 3 & 3 & 0 & 3 \\
\hline Management & 141 & 21 & 17 & 9 & 4 & 1 & 2 & 2 \\
\hline $\begin{array}{c}\text { Computer Science Theory } \\
\text { Methods }\end{array}$ & 94 & 6 & 9 & 1 & 0 & 1 & 0 & 1 \\
\hline Business & 73 & 7 & 3 & 5 & 3 & 0 & 1 & 0 \\
\hline $\begin{array}{l}\text { Computer Science } \\
\text { Interdisciplinary Application }\end{array}$ & 43 & 6 & 3 & 1 & 0 & 0 & 1 & 0 \\
\hline $\begin{array}{l}\text { Computer Science Software } \\
\text { Engineering }\end{array}$ & 45 & 4 & 0 & 0 & 1 & 0 & 0 & 1 \\
\hline $\begin{array}{l}\text { Engineering Electrical } \\
\text { Electronic }\end{array}$ & 40 & 4 & 3 & 2 & 0 & 0 & 1 & 0 \\
\hline $\begin{array}{l}\text { Operations Research } \\
\text { Management Science }\end{array}$ & 37 & 0 & 2 & 5 & 0 & 0 & 2 & 0 \\
\hline $\begin{array}{c}\text { Green Sustainable Science } \\
\text { Technology }\end{array}$ & 26 & 2 & 0 & 2 & 0 & 1 & 0 & 0 \\
\hline
\end{tabular}

Figure 3. Heat Map of Citations by Article and Citing Field (select articles only)

\section{SUMMARY AND CONCLUSIONS}

Our analysis indicates that there is significant heterogeneity among the analyzed articles in terms of defining the purposes of a literature review, but there is significant homogeneity among the guidelines provided by various authors regarding conducting the review. Most guidelines suggest that researchers should follow a structured approach to conducting reviews, starting with determining the focus and scope of the review, then developing criteria for literature search and inclusion, collecting and analyzing the relevant studies, developing a theoretical framework, and reporting the findings. The aim of all literature review guidelines we studied is to help other researchers produce a high-quality review paper. The quality of literature reviews is highly associated with the notion of rigor (Levy \& Ellis, 2006; Okoli, 2015; Bandara et al., 2015), which involves replicability, transparency, and unbiasedness of the review protocol. We find that the notion of "high quality" and the related idea of "rigor" evolve over time, first in the direction of specifying a very structured review process and then in the direction of relaxing or replacing some of the requirements in favor of producing a quality result. Early papers focus on the general structure of the review. To ensure rigor, more recent articles in our sample offer very specific guidelines for conducting certain stages of the review, such as the literature search or analysis. In contrast, other papers argue that the aim of the literature review is not to achieve rigor in the process but to develop and understand insights through continuous engagement with the reviewing process (Boell \& Cecez-Kecmanovic, 2014). To this end, papers devoted to specific methods, such as grounded theory or hermeneutics begin to appear. In addition, papers that question the strict adherence to a systematic protocol (Boell \& CecezKecmanovic, 2015) or inflexible classification schemes and reproducibility goals (Leidner, 2018) are also being published. We find that these developments are generating ongoing discussion in the IS field. For example, Boell \& Cecez-Kecmanovic's 2015 article led to several invited commentaries from other respected IS scholars. The topic of literature reviews continues to be actively debated in conferences panels featuring diverse perspectives on various guidelines and lessons learned while applying them (Schryen et al., 2017).

Our analysis of the impact of the selected literature review guidelines shows that despite the above-noted differences, these papers are regarded as containing benchmarks for doing systematic literature reviews in the IS field, as evidenced by high citation counts. Additionally, the fact that the number of citations per year increases with time for most papers and the fact that the IS guidelines are cited in a diversity of fields suggest the existence of a growing interest in conducting systematic literature reviews among scholars in many disciplines. This may be a sign that writing literature reviews is an increasingly important skill, as the papers that we reviewed argue, for any researcher. We hope our analysis can help guide researchers from IS and other fields to understand not just the general best practices for conducting literature reviews, but also the emerging options available for generating a quality result.

Future research could investigate the nature of citations to the literature review guidelines - namely whether the citing articles criticize the best practices or adopt them. In addition, it would be beneficial to understand how journal and conference reviewers perceive the literature review guidelines, and what expectations they have for well-executed papers submitted for review. 


\section{Issues in Information Systems}

Volume 20, Issue 3, pp. 83-93, 2019

\section{REFERENCES}

Bandara, W., Furtmueller, E., Gorbacheva, E., Miskon, S., \& Beekhuyzen, J. (2015). Achieving rigor in literature reviews: Insights from qualitative data analysis and tool-support. Communications of the Association for Information Systems, 37, 154-204.

Boell, S. K., \& Cecez-Kecmanovic, D. (2014). A hermeneutic approach for conducting literature reviews and literature searches. Communications of the Association for Information Systems, 34, 257-286.

Boell, S. K., \& Cecez-Kecmanovic, D. (2015). On being 'systematic' in literature reviews. Journal of Information Technology, 30, 161-173.

Kitchenham, B., Brereton, O. P., Budgen, D., Turner, M., Bailey, J., \& Linkman, S. (2009). Systematic literature reviews in software engineering-a systematic literature review. Information and Software Technology, $51(1), 7-15$.

Leidner, D. E. (2018). Review and theory symbiosis: An introspective retrospective. Journal of the Association for Information Systems, 19(6), 552-567.

Levy, Y., \& Ellis, T. J. (2006). A systems approach to conduct an effective literature review in support of information systems research. Informing Science, 9, 181-212.

Moher, D., Liberati, A., Tetzlaff, J., Altman, D. G., \& Prisma Group (2009). Preferred reporting items for systematic reviews and meta-analyses: the PRISMA statement. PLoS Medicine, 6(7).

Okoli, C. (2015). A guide to conducting a standalone systematic literature review. Communications of the Association for Information Systems, 37, 879-910.

Paré, G., Trudel, M. C., Jaana, M., \& Kitsiou, S (2015). Synthesizing information systems knowledge: A typology of literature reviews. Information \& Management, 52(2), 183-199.

Pickering, C. M., \& Byrne, J. (2014). The benefits of publishing systematic quantitative literature reviews for PhD candidates and other early career researchers. Higher Education Research and Development, 33, 534-548.

Rowe, F. (2014). What literature review is not: diversity, boundaries and recommendations. European Journal of Information Systems, 23(3), 241-255.

Schryen, G. (2015). Writing qualitative IS literature reviews-Guidelines for synthesis, interpretation and guidance of research. Communications of the Association for Information Systems, 37, 286-325.

Schryen, G., Benlian, A., Rowe, F., Gregor, S., Larsen, K., Petter, S., Paré, G., Wagner, G., Haag, S., \& Yasasin, E. (2017). Literature reviews in IS research: What can be learnt from the past and other fields? Communications of AIS, 41, 759-774.

Siddiqi, N. (2012). Credit risk scorecards: developing and implementing intelligent credit scoring. John Wiley \& Sons.

Templier, M., \& Paré, G. (2015). A framework for guiding and evaluating literature reviews. Communications of the Association for Information Systems, 37, 112-137.

Tranfield, D., Denyer, D., \& Smart, P. (2003). Towards a methodology for developing evidence-informed management knowledge by means of systematic review. British Journal of Management, 14(3), 207-222.

Webster, J., \& Watson, R. T. (2002). Analyzing the past to prepare for the future: Writing a literature review. MIS Quarterly, 26(2), xiii-xxiii.

Wolfswinkel, J. F., Furtmueller, E., \& Wilderom, C. P. (2013). Using grounded theory as a method for rigorously reviewing literature. European Journal of Information Systems, 22(1), 45-55. 
Issues in Information Systems

Volume 20, Issue 3, pp. 83-93, 2019

\section{ACKNOWLEDGEMENTS}

The paper is based on work performed by all the authors during a Bentley University doctoral course taught by the last author (Alina Chircu). All authors contributed equally to the paper. The author names for all but the last author are listed in alphabetical order 